\title{
Noise Minimization for Ultra-High SNR Class-D Power Amplifiers
}

\author{
Mario Mauerer and Johann W. Kolar
}

\begin{abstract}
Important noise sources affecting power output waveforms of digitally controlled switch-mode (Class-D) converters/amplifiers are investigated with a $400 \mathrm{~V}$ hardware demonstrator utilizing gallium nitride (GaN) power transistors. First, the influence of the open-loop gains of the cascaded feedback control system on the load current signal-to-noise ratio (SNR), which is a key metric in low-noise, nanometer-precision mechatronic positioning applications, is demonstrated. With a PWM frequency of $200 \mathrm{kHz}$, the high achievable controller gains enable unprecedented SNR values in excess of $105 \mathrm{~dB}$ (DC-10 kHz). Next, it is shown how a real-time Kalman filter, calculated at a rate of $100 \mathrm{kHz}$ and used to attenuate sensor noise, increases the amplifier output SNR by more than $10 \mathrm{~dB}$. Furthermore, digital delta-sigma (noise shaping) modulation improves the SNR by $5 \mathrm{~dB}$, while not affecting distortion.
\end{abstract}

Index Terms - Delta-sigma modulation, feedback control, Kalman filter, low noise, power amplifier, pulse-width modulation, signal-to-noise ratio (SNR), wide-bandgap semiconductors.

\section{INTRODUCTION}

$\mathrm{P}$ OWER amplifiers are universally employed in applications such as high-frequency mobile base stations, consumer audio or magnetic resonance imaging systems, with output power levels covering more than six orders of magnitude. This work investigates low-noise (microampere), high-power (kilowatt) amplifiers indispensable for industrial nanoscale positioning applications, where the amplifiers are embodied as power electronics converters that provide amplified output waveforms which should follow the corresponding reference signals with minimum deviation and should show a very low noise level in wide frequency ranges.

The considered amplifiers generate well-controlled output currents that produce torques and forces in different types of electromagnetic actuators such as magnetic bearings or single-/ three-phase permanent magnet motors. Vibration isolation systems, for example, are often used to decouple sensitive positioning stages from ground vibrations caused by nearby equipment or geologic activity [1], [2]. Consequently, their actuators require current signals of extremely low noise in order to prevent the generation of undesired movements. Positioning stages used for lithography scanners or back-end processes in integrated circuit manufacturing often feature magnetic or

Manuscript received May 15, 2018

The authors are with the Power Electronic Systems Laboratory, ETH Zurich, Switzerland (e-mail: mauerer@lem.ee.ethz.ch).

Digital Object Identifier 10.24295/CPSSTPEA.2018.00033 air bearings, whose key characteristics are low friction and damping [3]-[6]. Consequently, the noise requirements for the driving amplifiers are critical in a frequency range from DC to $\approx 10 \mathrm{kHz}$, as the mechanical stages typically provide little attenuation in the transfer functions from actuator currents to positioning stage accelerations in this bandwidth. Besides very low-noise, amplifiers in lithography systems also have to provide high output powers and dynamics to enable fast accelerations of the motion stages in order to sustain high production throughput rates and large wafer diameters [7]. Due to integrated semiconductor features that continuously shrink in size and grow in complexity, actuator load current dynamic ranges in excess of $90 \mathrm{~dB}$ are expected, with signal-to-noise ratio (SNR) requirements in excess of $100 \mathrm{~dB}$ [8], [9].

Traditionally, linear amplifiers, which are commonly based on power operational amplifiers or discrete devices, are preferred for these tasks due to their inherently low noise over wide frequency ranges, combined with low output impedances [10]. Noise and linearity can be further improved with closedloop feedback systems that can be implemented in the analog or digital domain, whereas the former also features intrinsic low noise due to the absence of (digital) amplitude quantization [11], [12]. However, linear amplifiers collectively suffer from limited efficiencies and limited output power levels. Hybrid amplifiers strive to alleviate these restrictions by combining linear amplifiers with high-power, efficient switch-mode converters [13], [14]. Nonetheless, such topologies increase the complexity of the electrical circuit and, if utilized, the feedback control system, as the fundamentally different characteristics of the linear and switched conversion stages, with respect to their dynamics and input/output impedances, have to be analyzed and stabilized individually, which increases development effort and cost.

Therefore, this work analyzes key influence factors on the output noise of digitally controlled, pulse-width modulated (PWM) switch-mode power amplifiers/converters. The analysis is similar to the one conducted in [15], which focuses on distortion and utilizes the same hardware demonstrator system to provide verification measurements as in this work. Fig. 1 lists important sources of noise in such converters. Due to the intended application in mechatronic positioning systems, the amplifier output current noise is considered, as the generated electromagnetic actuator forces are proportional to current. Nonetheless, the presented insights are universally applicable to amplifiers that provide either a controlled current or a controlled output voltage. Throughout this work, the term noise encompasses all undesired signal components, except for 


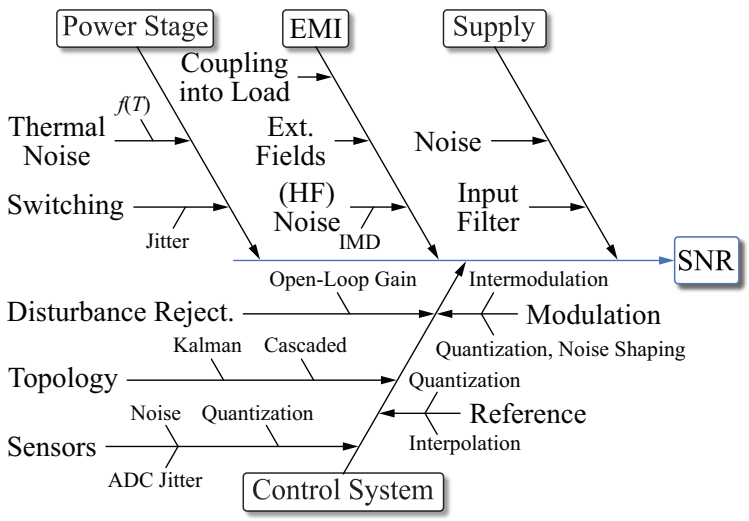

Fig. 1. Nonidealities of system components influencing the signal-to-noise ratio (SNR) of the output quantities of digitally controlled switch-mode power amplifiers.

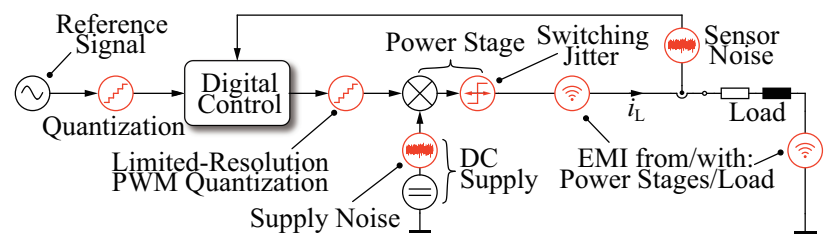

Fig. 2. Amplifier noise sources can be modeled as series-connected voltage/signal sources. Reference signal quantization noise is effectively eliminated with digital signals of sufficient resolution $(>30$ bit). Electromagnetic interference (EMI) within the amplifier itself (e.g., from other switching stages or auxiliary $\mathrm{DC} / \mathrm{DC}$ regulators) is rejected with shielding and proper $\mathrm{PCB}$ layout techniques.

harmonics in AC output signals (that are caused by amplifier nonlinearities). Thus, signals with spectral components at $50 \mathrm{~Hz}$, e.g., due to electromagnetic interference (EMI) originating from the power grid, are also accounted to the noise power [16].

Fig. 2 outlines key noise sources in the context of a switchmode power amplifier, modeled as series-connected voltage or (digital) signal sources. The quantization noise introduced by the limited-resolution PWM [17], as well as the timedomain jitter of the power transistor switching actions [18], are linked to stochastic processes and thus introduce wideband noise of uniform power density [19]. Supply noise, or electromagnetic interference (EMI) from external sources by means of stray fields that couple to the amplifier's circuit, on the other hand, often exhibit spectral components at distinct frequencies. Due to nonlinear elements within the amplifier, such as half-bridges with dead time or saturating inductors, these frequency components can undergo intermodulation distortion, which results in noise signals at lower frequencies [20], [21]. Therefore, it is important to also consider noise sources outside the frequency band of interest (DC to $10 \mathrm{kHz}$ in this work), as their intermodulation products can appear in sensitive frequency ranges. In this work, it is demonstrated to what amount the mentioned noise sources influence the SNR of the load current, which is an important quality measure for low-noise amplifiers. Note that fundamental output load current frequencies of amplifiers in mechatronic motion systems range from DC to $\approx 200 \mathrm{~Hz}$.

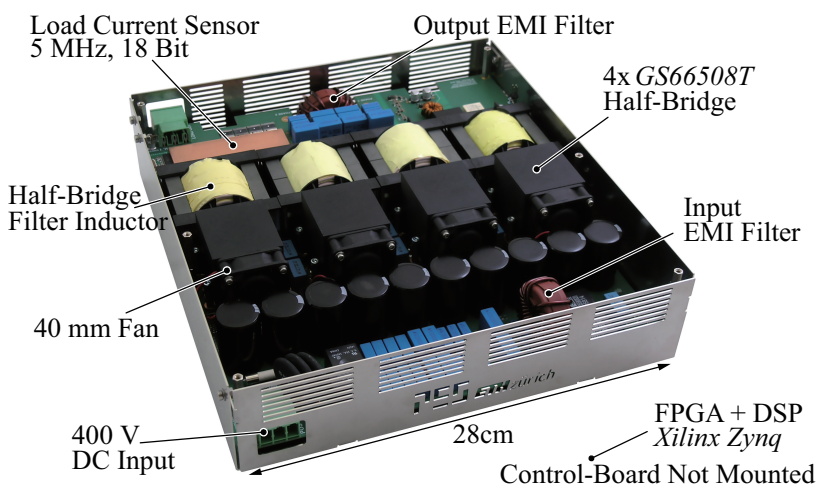

Fig. 3. Hardware demonstrator featuring four GaN Systems GS66508T halfbridges $(650 \mathrm{~V}, 30 \mathrm{~A})$ and high-resolution current/voltage ADC systems. The max. DC-link voltage is $400 \mathrm{~V}$. The system is designed for supplying a singlephase inductive-resistive load with a maximum current amplitude of $25 \mathrm{~A}$. The control board is mounted on top (not shown) and features a high-performance FPGA and DSP to implement complex control structures such as Kalman filters.

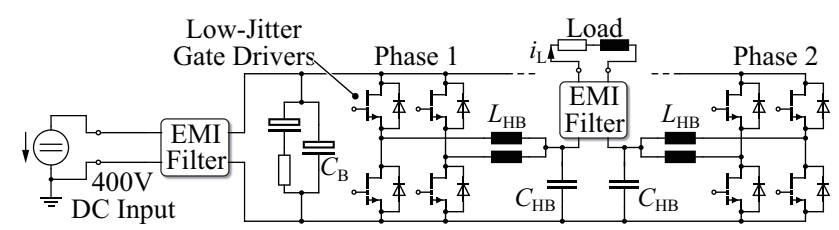

Fig. 4. Power circuit topology of the hardware demonstrator. The bridgetied, single-phase load is supplied by two interleaved half-bridges, which can implement two power stage topologies, namely the regular interleaving of two half-bridges and the dual-buck topology. EMI filters ensure compliance with corresponding standards and reduce EMI for sensitive nearby equipment of the positioning systems. $L_{\mathrm{HB}}=700 \mu \mathrm{H}, C_{\mathrm{HB}}=12 \mu \mathrm{F}$.

To conduct this analysis, a hardware demonstrator, illustrated in Fig. 3, is employed. It features four interleaved half-bridges based on $650 \mathrm{~V}$ enhancement-mode, high-electron-mobility gallium nitride ( $\mathrm{GaN}$ ) power transistors ( $\mathrm{GaN}$ Systems, GS66508T), which enables the system to deliver up to $25 \mathrm{~A}$ (peak) to a bridgetied, single-phase load. The nominal DC-link voltage is $400 \mathrm{~V}$ and the PWM pulse repetition frequency $f_{\mathrm{PWM}}$ is $\leqslant 200 \mathrm{kHz}$, limited by transistor switching losses. The converter's power circuit topology is depicted in Fig. 4. Further details of this system are given in [15], which focuses on the output distortion of the amplifier.

To eliminate time-domain jitter from the half-bridge switching transitions, which is a considerable source of wideband noise, low-jitter gate drivers, as presented in [18], are used to drive all power transistors. The output noise of the limitedresolution pulse-width modulators, which are a considerable source of wideband noise, is reduced with digital delta-sigma modulators/noise shapers (NS), which can shift the PWM quantization noise to higher frequencies, as shown in [17] and in Section IV-A below.

The interleaved half-bridges enable the implementation of two distinct power stages, namely the regular interleaved halfbridge arrangement and the dual buck topology, whereas the latter is not subject to distortion related to half-bridge interlock (dead time) intervals, as demonstrated in [15], which also provides details of the demonstrator's closed-loop feedback 


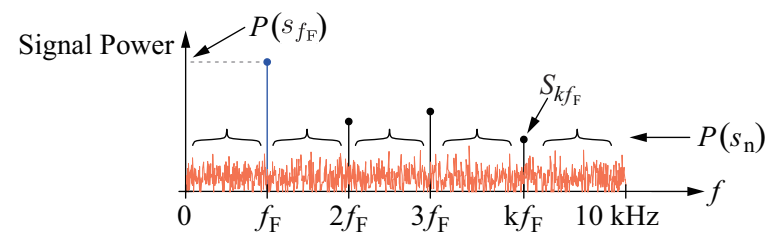

Fig. 5. Noise power considered for the SNR excludes the power of the signal at the fundamental frequency $f_{\mathrm{F}}$ and its harmonics. The frequency band from DC to $10 \mathrm{kHz}$ is critical for mechatronic motion systems.

control system.

To increase the control system's flexibility and to de-couple the cascaded control loops dynamically, each system state, i.e., the four half-bridge currents, the two phase voltages and the load current, are measured. As shown in this work, sensor noise critically affects the control system and hence, low-noise voltage and current sensors are used, featuring 16-bit analog-to-digital converters (ADCs) that operate without anti-aliasing filters (for increased control system bandwidth) and synchronously with the converter's switching actions, in conjunction with optimized signal processing circuits (filters/amplifiers) [22]. The load current sensor employs an 18-bit ADC with an antialiasing filter (it samples asynchronously to the converter's switching instants) and a sampling rate of $5 \mathrm{MHz}$ to make use of oversampling, which reduces ADC quantization noise [22]. Further details of the sensor systems are given in [15]. An FPGA is well-suited to acquire and process the high-speed, high-resolution data streams from the individual ADCs, which results in an overall data rate of $112 \mathrm{Mbit} \mathrm{s}^{-1}$ when operating with $f_{\mathrm{PWM}}=200 \mathrm{kHz}$. However, some control tasks, like a Kalman filter, are implemented with low complexity as a floating-point accurate, procedural program. Consequently, the converter's processing system features a Xilinx Zynq Z-7020, which combines an FPGA with two $666 \mathrm{MHz}$ ARM Cortex-A9 CPUs and high-performance floating-point units [23].

For the calculation of the load current SNR, the noise of the current signal is considered from DC to $10 \mathrm{kHz}$, as this frequency range is critical for precision positioning applications:

$$
S N R=10 \log _{10} \frac{P\left(s_{f_{F}}\right)}{P\left(s_{n}\right)},
$$

where $P\left(s_{f_{\mathrm{F}}}\right)$ is the power of the desired amplifier output signal component $s_{f_{\mathrm{F}}}$ with fundamental frequency $f_{\mathrm{F}}$ (assuming a sinusoidal signal), and $P\left(s_{n}\right)$ is the power of the noise in a frequency range from $\mathrm{DC}$ to $10 \mathrm{kHz}$, which considers the power of all signal components in this bandwidth, except the power of the fundamental component at $s_{f_{\mathrm{F}}}$ and its integer harmonics $s_{k f_{\mathrm{F}}}$, with $k \in$ $[1,2, \ldots][20]$, as shown with the following equation and Fig. 5:

$$
P\left(s_{n}\right)=\int_{D C}^{10 \mathrm{kHz}} P(s) \mathrm{d} f-\sum_{k=1}^{\infty} P\left(s_{k f_{\mathrm{F}}}\right) .
$$

As it will be demonstrated in this work, the noise power of the output signal is independent of the fundamental frequency $f_{\mathrm{F}}$ and, to a large extent, also from the amplitude of $s_{f_{\mathrm{F}}}$.

The presented demonstrator is capable of providing a lownoise output load current $i_{\mathrm{L}}$ and consequently, an independent reference current sensor with sufficiently low inherent measurement noise is required for the acquisition of verification measurements. For this purpose, a LEM IT 65-S current sensor, in conjunction with a high-precision, low-noise spectrum analyzer, is used to acquire the sinusoidal amplifier output current [15], [24], [25]. This setup is capable of measuring SNR values (according to (1)) in excess of $\approx 108 \mathrm{~dB}$. Amplifier output current fundamental frequencies $f_{\mathrm{F}}$ of $35 \mathrm{~Hz}$ and $210 \mathrm{~Hz}$ are used, which are similar to actuator frequencies in mechatronic positioning systems, and whose harmonics do not interfere with harmonics of the power grid, as described in [15]. The amplifier generates sinusoidal output currents, as this waveform allows the effective analysis of SNR and distortion, and is common in mechatronic motion control systems. The utilized resistive-inductive passive loads comprise highpower thick-film resistors and high-current inductors, whereas three different loads, which recreate the characteristics of different mechatronic actuators (e.g., linear motors with and without iron cores), are employed for the measurements: $2 \Omega+10 \mathrm{mH}, 5 \Omega+2.5 \mathrm{mH}$ and $10 \Omega+100 \mu \mathrm{H}$. The $100 \mu \mathrm{H}$ inductor employs a gapped ferrite core, whereas the two other inductors are significantly larger and their cores are made from laminated silicon steel (designed for low-frequency power grid applications).

Several key contributions arise from this work. First, stateof-the-art, high-performance signal processors enable highfrequency and high-gain feedback controllers that can provide a substantial disturbance attenuation. Measurements directly compare the achievable performance, which is strongly affected by the PWM frequency. Different influence factors, such as load impedance or DC-supply noise, are highlighted, and the limits to the achievable load current SNR are demonstrated. Detailed measurements are performed to isolate the effects of different noise sources by, e.g. utilizing a battery supply. Next, a real-time Kalman filter implementation is presented in detail. Measurements demonstrate its value for precision switchmode amplifiers, and design trade-offs are outlined. Finally, the influence of noise shaping PWM modulators on a highgain closed-loop amplifier is demonstrated with measurements. Hence, this work provides comprehensive sensitivity analyses of amplifier subsystems with respect to noise, which facilitates future design decisions.

In the following, Section II investigates the influence of the control system's disturbance rejection capability on the amplifier's load current SNR. Next, Section III presents the effects of a Kalman filter, which reduces residual sensor noise. Section IV analyzes the noise contributions from the PWM and the noise shapers under closed-loop operation, as well as the influence on SNR when utilizing the low-distortion dual buck topology. Section V summarizes the results of this work and provides an outlook.

\section{Control System}

A closed-loop feedback control system is an essential con- 


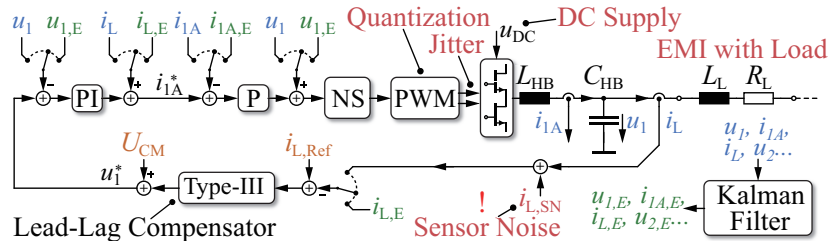

Fig. 6. Simplified diagram of the cascaded control system (second bridge leg (phase) and second half-bridge of the shown bridge leg omitted). Important sources of noise are outlined (sensor noise only shown for $i_{\mathrm{L}}$, despite all measurements being subject to noise).

tribution for high-performance output waveforms of any amplifier system, as it is capable of significantly rejecting and/or attenuating disturbances such as noise or harmonic distortion.

Fig. 6 illustrates the simplified block diagram of the demonstrator's decoupled, closed-loop cascaded feedback control system (redundant elements like the second phase and the interleaved half-bridge are omitted for simplicity; see [15] for more details). Dominant sources of noise are illustrated in red. Note that the sensor noise coupling is only shown for the load current measurement $i_{\mathrm{L}}$, despite all measurements being subject to sensor noise, caused by analog filters or amplifiers, and the subsequent ADC stages [22].

The four half-bridge currents are controlled by individual proportional (P) controllers, which obtain their reference signals from the proportional-integral (PI) phase-voltage controllers. A type-III (lead-lag) compensator controls the load current $i_{\mathrm{L}}$. All controllers are digital and implemented in the system's FPGA, and are calculated synchronously with the converter's PWM, at the same frequency. They are tuned using loop shaping, with consideration of the delays caused by the calculation times of the control algorithms and the sampling of the PWM, which reduces the phase margins of the control loops. All open-loop gains are maximized, while maintaining phase margins of $50^{\circ}$. More details are given in [15]. A Kalman filter (see Section III below) can be utilized to provide low-noise estimates of the sensor measurements.

Sources of noise that act on the plants of the dynamic system (e.g., the supply noise) appear as disturbances to the control system and are thus effectively attenuated with the open-loop gains of the feedback loops [26]-[28]. Harmonic distortion is also categorized as such a disturbance and the influence of the closed-loop controllers on the amplifier's linearity is discussed in [15]. This disturbance rejection is an important feature of closed-loop feedback control systems. They are thus a key contributor to low-noise and lowdistortion power conversion systems. On the other hand, the control system reacts equally sensitive to sensor noise than to its reference input and consequently, it cannot be rejected by feedback control, which constitutes a fundamental disadvantage [26], [28]. This corroborates the necessity for low-noise sensors or advanced control methods, such as a Kalman filter.

Nonetheless, a high disturbance rejection is still desired to attenuate sources of noise, to linearize the amplifier and to

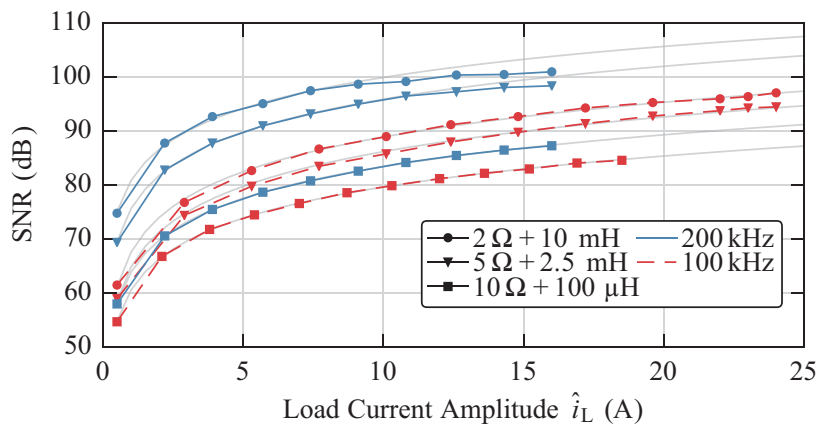

Fig. 7. SNR measured for different loads at different $f_{\mathrm{PWM}}$, which correlates with the achievable disturbance rejection performance of the controllers. Kalman filter disabled (cf. Section III). At high currents and high SNR, noise from the DC supply affects SNR unfavorably. Grey lines are individual least-squares fits for each amplifier configuration with: $\mathrm{SNR}(\mathrm{dB})=c_{0} \ln \left(\hat{i}_{\mathrm{L}}\right)+c_{1} \cdot u_{\mathrm{DC}}=400 \mathrm{~V}$, $f_{\mathrm{F}}=35 \mathrm{~Hz}$.

improve its reference tracking performance. This demands high open-loop gains of all feedback loops, which are limited by their respective stability margins.

\section{A. Load Current Noise Measurements}

As mentioned above and as further discussed in [15], the control performance increases with the PWM frequency of the converter, as delays are reduced. Fig. 7 illustrates this with measurements of the amplifier's load current SNR, obtained at two different PWM switching frequencies. Due to the increased switching losses of the power transistors at higher $f_{\text {PWM }}$, the achievable $\hat{i}_{\mathrm{L}}$ is reduced, as derived in [15]. The power dissipation capability of the $10 \Omega$ load resistor, and the utilized DC power supply, may also limit $\hat{i}_{\mathrm{L}}$ in certain measurement configurations. As expected, the control topology with the higher open-loop gains rejects noisy disturbances better and hence, SNR is improved by up to $10 \mathrm{~dB}$. Naturally, load configurations with higher inductance values show less current noise due to the fact that the power amplifier operates as a voltage source, whose noise components translate to noise currents in accordance with the load impedance.

The gray lines in Fig. 7 are logarithmic least-squares fits of the SNR values of each corresponding amplifier configuration (the formula is given in the figure caption). As given by (1), the SNR exhibits a logarithmic behavior as a function of the load current, if the noise power remains constant. This is behavior of the SNR is experienced with the measurements in Fig. 7, which hence implies that the load current noise is not affected by the magnitude of $\hat{i}_{\mathrm{L}}$. This is expected, as major noise sources, e.g., the PWM or gate drive signal isolation, operate independently of the output current amplitude [18]. Consequently, the achievable SNR is limited by the load current capability of the power amplifier, as the noise power remains, ideally, constant. However, at increased load currents, the SNR measurements can deviate from their predicted values (cf. the $2 \Omega+10 \mathrm{mH}$ load in Fig. 7). This is attributed to the fact that noise originating from the DC laboratory power supply increases at high output powers.

The deteriorating effects of increasing DC supply noise 


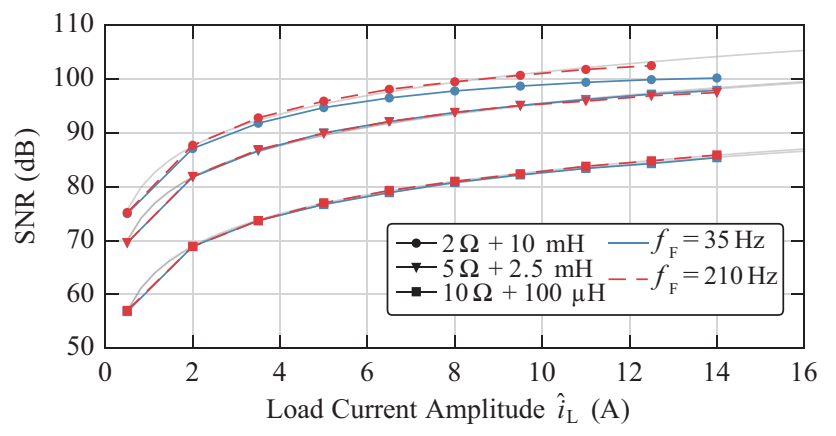

Fig. 8. SNR measured for different loads and fundamental frequencies $f_{\mathrm{F}}$. With higher power ripple frequencies, the DC supply can provide better voltage regulation and thus, less output noise. Regular cascaded control system (Kalman filter disabled). $u_{\mathrm{DC}}=400 \mathrm{~V}, f_{\mathrm{PWM}}=200 \mathrm{kHz}$

is also visible in the current amplitude sweeps shown in Fig. 8, which shows similar measurements, but performed at two different fundamental load current frequencies $f_{\mathrm{F}}$. The degrading effect on the SNR is, again, only visible with the $2 \Omega+10 \mathrm{mH}$ load and for $f_{\mathrm{F}}=35 \mathrm{~Hz}$, whereas the measurement performed with $f_{\mathrm{F}}=210 \mathrm{~Hz}$ follows its expectation well (i.e., it does not derive from its logarithmic fit line). This distinction of load current SNR between the different fundamental frequencies is owed to the fact that the amplifier's DC-link buffer capacitors $\left(C_{\mathrm{B}}=1.8 \mathrm{mF}\right.$ in the demonstrator system) present less impedance for the singlephase load ripple current at higher $f_{\mathrm{F}}$ and consequently, it is shunted from the DC-link supply, whose output current is thus experiencing less variation. This improves its voltage regulation and hence, noise is reduced. The DC-link voltage could be measured and used in conjunction with a feedforward compensation method to mitigate the impacts of the DC-link voltage variations. However, as shown in [15], this approach can potentially introduce further noise and thus, deteriorate the SNR.

Generally, however, the SNR is independent of $f_{\mathrm{F}}$, which is expected as the disturbance rejection of the feedback controllers is not affected by the frequency of their reference signals. This is not the case for harmonic distortion as shown in [15], as the control system provides less open-loop gain at higher frequencies, which is needed to attenuate the harmonics at integer multiples of $f_{\mathrm{F}}$.

To further illustrate the influence of the DC supply on the load current noise, Fig. 9 shows measurements where the amplifier output SNR, when operating with a switch-mode laboratory supply, is compared to the performance with a battery supply, comprising six individual, series-connected $12 \mathrm{~V}$ lead-acid cells that typically feature a negligible voltage noise. For this measurement, the DC-link voltage is set to $\approx 75 \mathrm{~V}$ for both supply types. By eliminating the noise of the switch-mode laboratory supply, the load current SNR improves slightly and does not deteriorate at higher load currents.

These measurements emphasize the requirement for lownoise DC supply voltage sources, despite the usage of highgain feedback controllers. Amplifier operation with single-

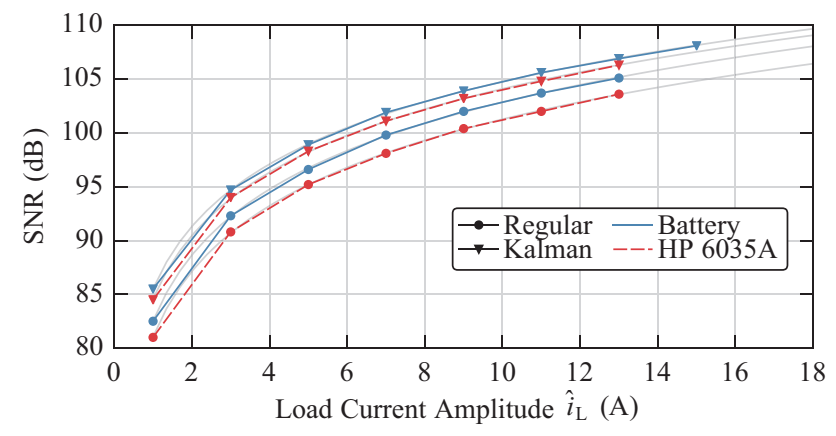

Fig. 9. Load current SNR improves with a battery supply compared to a switchmode, high-power laboratory supply (HP 6035A). Regular cascaded control system executed with $200 \mathrm{kHz}$, Kalman controller executed with $100 \mathrm{kHz}$ (cf. Section III). $f_{\mathrm{PWM}}=200 \mathrm{kHz} . u_{\mathrm{DC}}=75 \mathrm{~V}, f_{\mathrm{F}}=35 \mathrm{~Hz}$. Load: $2 \Omega+10 \mathrm{mH}$.

phase loads are characterized by a large power pulsation, which aggravates the stability of the DC supply further. In case of a switch-mode supply system, a good rejection of signal components at the supply's switching frequency is also desired, as intermodulation distortion can shift such components into sensitive frequency ranges.

In the following, sensor noise, which is a critical influence factor, is largely eliminated by employing a Kalman filter.

\section{Real-Time Kalman Filter}

A fundamental impediment of closed-loop feedback systems is their high sensor noise sensitivity, as this noise source is essentially amplified by the (usually) high controller gains [26], [28]. Despite the low-noise voltage and current sensors in the demonstrator system [22], sensor noise can still not be neglected, as it will be shown in this section.

Generally, the Kalman filter presents an approach to provide estimates of unknown or noisy states of a dynamic system (e.g., voltage, current, position, speed etc.), given some knowledge about the dynamic system itself, and usually (noisy) measurements of some (or all) states [29]. Kalman filters, which can manifest in different implementations that are often tailored for specific applications (e.g., with linear or nonlinear dynamic system models), are commonly used in guidance or navigation. Such applications do not require high execution rates of the required calculations due to their limited dynamics. However, as shown in Section II and [15], the controller execution rate is critical for achieving amplifier output waveforms of low noise and distortion. In the discussed application, the Kalman filter is used to attenuate sensor noise and hence, it must be computed with the same rate as the feedback control system (i.e., in realtime), in order to provide the updated low-noise estimates of the measurements for each control cycle.

Due to their computational complexity, real-time Kalman filters in power electronic applications usually do not exceed execution rates in excess of $5 \mathrm{kHz}$ to $30 \mathrm{kHz}$, which is insufficient for precision amplifiers due to the accompanying reduction of achievable control gain and/or bandwidth [30][34]. By implementing the Kalman algorithm using con- 


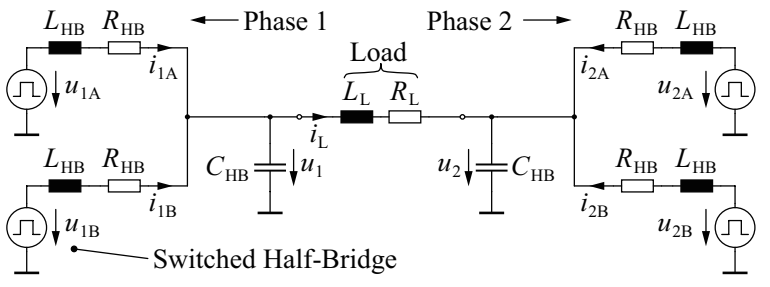

Fig. 10. Linear dynamic system model of the demonstrator system power stage used for the Kalman filter. $L_{\mathrm{HB}}=700 \mu \mathrm{H}, R_{\mathrm{HB}}=70 \mathrm{~m} \Omega, C_{\mathrm{HB}}=12 \mu \mathrm{F}$.

figurable logic (i.e., with an FPGA), the execution rates can be increased to more than $150 \mathrm{kHz}$ [35], [36]. However, this comes at the cost of a significantly increased implementation complexity and a loss of design flexibility. Consequently, this work presents a real-time Kalman filter implementation, executed as a procedural program that is calculated by a conventional processor. It is part of the demonstrator's feedback control system and it can provide low-noise estimates of the sensor data at a rate of $100 \mathrm{kHz}$, while featuring seven system states and four inputs in its underlying dynamic model, as presented in the following.

The Kalman algorithm requires a dynamic model of the physical system. In order to reduce the computational complexity, a linear model of the power amplifier system, illustrated in Fig. 10, is utilized. The switched half-bridges are modeled as controlled voltage sources to obtain a linear system. Its description, including the state vector $x$, the input vector $u$, and a vector $w$ that models noise, can be given by

$$
\dot{x}=A x+B u+G w,
$$

with the state and input vectors as follows:

$$
\begin{gathered}
x=\left[\begin{array}{ccccccc}
i_{1 \mathrm{~A}} & i_{1 \mathrm{~B}} & i_{2 \mathrm{~A}} & i_{2 \mathrm{~B}} & i_{\mathrm{L}} & u_{1} & u_{2}
\end{array}\right]^{\top}, \\
u=\left[\begin{array}{lllll}
u_{1 \mathrm{~A}} & u_{1 \mathrm{~B}} & u_{2 \mathrm{~A}} & u_{2 \mathrm{~B}}
\end{array}\right]^{\top},
\end{gathered}
$$

and the system matrices as:

$$
A=\left[\begin{array}{ccccccc}
\frac{-R_{\mathrm{HB}}}{L_{\mathrm{HB}}} & 0 & 0 & 0 & 0 & \frac{-1}{L_{\mathrm{HB}}} & 0 \\
0 & \frac{-R_{\mathrm{HB}}}{L_{\mathrm{HB}}} & 0 & 0 & 0 & \frac{-1}{L_{\mathrm{HB}}} & 0 \\
0 & 0 & \frac{-R_{\mathrm{HB}}}{L_{\mathrm{HB}}} & 0 & 0 & 0 & \frac{-1}{L_{\mathrm{HB}}} \\
0 & 0 & 0 & \frac{-R_{\mathrm{HB}}}{L_{\mathrm{HB}}} & 0 & 0 & \frac{-1}{L_{\mathrm{HB}}} \\
0 & 0 & 0 & 0 & \frac{-R_{\mathrm{L}}}{L_{\mathrm{L}}} & \frac{1}{L_{\mathrm{L}}} & \frac{-1}{L_{\mathrm{L}}} \\
\frac{1}{C_{\mathrm{HB}}} & \frac{1}{C_{\mathrm{HB}}} & 0 & 0 & \frac{-1}{C_{\mathrm{HB}}} & 0 & 0 \\
0 & 0 & \frac{1}{C_{\mathrm{HB}}} & \frac{1}{C_{\mathrm{HB}}} & \frac{1}{C_{\mathrm{HB}}} & 0 & 0
\end{array}\right],
$$

$$
B=\left[\begin{array}{cccc}
\frac{1}{L_{\mathrm{HB}}} & 0 & 0 & 0 \\
0 & \frac{1}{L_{\mathrm{HB}}} & 0 & 0 \\
0 & 0 & \frac{1}{L_{\mathrm{HB}}} & 0 \\
0 & 0 & 0 & \frac{1}{L_{\mathrm{HB}}} \\
& O_{3 \times 4} & &
\end{array}\right] \text {, }
$$

with $O$ being the zero matrix where its indices define the number of its rows and columns. The state vector $x$ includes all capacitor/inductor voltages and currents, and the input vector $u$ contains the four half-bridge output voltages. In the demonstrator system, all seven states are measured by sensors and consequently, the measurement equation, including the measurement noise term $v$, is given by

$$
y_{m}=I x+v,
$$

with $I$ being the identity matrix. Noise is described by the process noise $w$, which accounts for sources of noise within the dynamic system, and measurement noise $v$, generated by the sensors. The noise is assumed to have a Gaussian probability distribution and a white (i.e., uniform) power spectral density. The corresponding noise covariance matrices $Q$ and $R$ are given as $Q=E\left(w w^{\top}\right)$ and $R=E\left(v v^{\top}\right)$, where $E$ is the expected value.

The process noise model is based on the assumption that the four half-bridge voltages are the only sources of noise within the dynamic system (apart from the measurement noise). This is given in the demonstrator hardware, in which the quantization noise of the limited-resolution PWM, or the half-bridge switching jitter, are injecting such process noise into the plant [17]. Other sources of noise within the system, such as EMI with the load (cf. Fig. 2), are neglected due to their comparably small noise powers and often parasitic natures. Consequently, $G$ is modeled such that the process noise only affects the four half-bridge currents:

$$
G=\left[\begin{array}{c|c}
I_{4 \times 4} & O_{4 \times 3} \\
\hline O_{3 \times 4} & O_{3 \times 3}
\end{array}\right] .
$$

The process noise (co)variances are modeled as

$$
Q=\left[\begin{array}{rrrr|r}
\sigma_{\mathrm{HB}}^{2} & 0 & 0 & 0 & \\
0 & \sigma_{\mathrm{HB}}^{2} & 0 & 0 & O_{4 \times 3} \\
0 & 0 & \sigma_{\mathrm{HB}}^{2} & 0 & \\
0 & 0 & 0 & \sigma_{\mathrm{HB}}^{2} & \\
\hline \multicolumn{7}{c}{O_{3 \times 4}} & & & O_{3 \times 3}
\end{array}\right],
$$

with $\sigma_{\mathrm{HB}}^{2}$ being the noise variance of the half-bridge voltages. 
Similarly, the measurement noise covariance matrix is

$$
R=\operatorname{diag}\left(\left[\sigma_{i_{\mathrm{HB}}}^{2} \sigma_{i_{\mathrm{HB}}}^{2} \sigma_{i_{\mathrm{HB}}}^{2} \sigma_{i_{\mathrm{HB}}}^{2} \sigma_{i_{\mathrm{L}}}^{2} \sigma_{u_{\mathrm{HB}}}^{2} \sigma_{u_{\mathrm{HB}}}^{2}\right]\right),
$$

which incorporates the variances of the three different types of sensors (half-bridge currents, load current and phase voltages). The diagonal matrix reflects the independence of the sensor noise sources, as each sensor is a self-contained unit which cannot influence the noise of other sensors. The corresponding sensor noise standard deviations, measured during converter operation with no applied voltages or currents, are given as $\sigma_{i_{\mathrm{HB}}}=2.0 \mathrm{~mA}, \sigma_{i_{\mathrm{L}}}=83.0 \mu \mathrm{A}$ and $\sigma_{u_{\mathrm{HB}}}=25.0 \mathrm{mV}$. For each measurement, $5 \times 10^{6}$ samples are obtained. As shown in [22], the sensor noise levels remain constant in their operating ranges. The low value for $\sigma_{i_{\mathrm{L}}}$ reflects the effort to create a low-noise measurement for the load current $i_{\mathrm{L}}$ (i.e., oversampled, 18-bit ADC with optimized analog processing circuitry) [22].

Using the presented system and noise covariance matrices (i.e., $A, B, G, Q$ and $R$ ), the Kalman filter can be designed. To reduce the required computations during converter operation and thus, to maximize the Kalman filter execution rate, a steady-state implementation of the Kalman estimation method is chosen. It is based on the assumption of constant noise covariance matrices $(Q, R)$ and hence, does not require the time-consuming calculation of inverse matrices or matrix factorizations [29]. This assumption holds in the considered power converter, as sensor noise and the system's input noise sources (e.g., PWM or half-bridge switching jitter) maintain a constant and independent noise behavior during operation.

In the presented application, the Kalman filter is implemented in a sampled system and consequently, the discretetime process noise covariance matrix is determined using Van Loan's method [29], whereas the continuous-time system description can be converted to a sampled model using common discretization methods such as zero-order hold or Tustin's method. The design process is well documented and common engineering tools can be used to effortlessly determine the optimal solution of the Kalman estimation problem [29], [37].

For the case of the considered steady-state Kalman filter, the algorithm, which provides the required low-noise measurement estimates, reduces to the calculation of a dynamic system of the following form, where the filtered measurements at each execution step $n$ are given by $y_{e}$, and $x_{n}$ is an internal state that is continuously updated:

$$
\begin{gathered}
x_{n}=A_{k} x_{n-1}+B_{k}\left[\begin{array}{l}
u_{n-1} \\
y_{m, n}
\end{array}\right], \\
y_{e}=C_{k} x_{n}+D_{k}\left[\begin{array}{l}
u_{n-1} \\
y_{m, n}
\end{array}\right] .
\end{gathered}
$$

Note that the solution of the Kalman estimation problem is embedded in the matrices $A_{k}, B_{k}, C_{k}$ and $D_{k}$ [37]. The

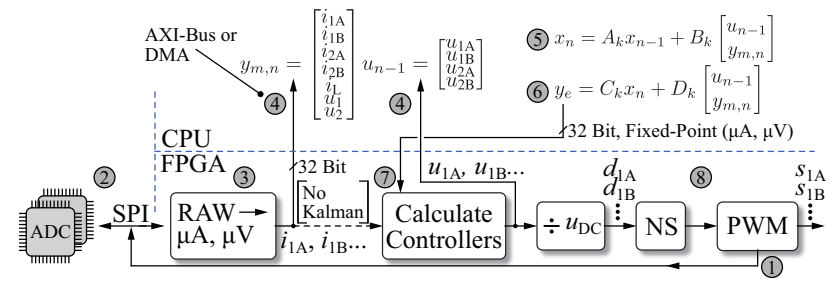

(1) New PWM Cycle (2) All ADCs Acquire Data (3) Data Converted to $\mu \mathrm{A}, \mu \mathrm{V}$ (4) Interrupt and Data Transfer to CPU $\quad$ (5) (6) Calculate Kalman Filter (7) Transfer of Estimates (8) Controllers, Noise Shapers, PWM Executed

Fig. 11. Data paths for the calculation of the steady-state Kalman filter in the demonstrator system. The entire algorithm can be executed with $100 \mathrm{kHz}$ (two PWM cycles if $f_{\mathrm{PWM}}=200 \mathrm{kHz}$ ). If the Kalman filter is not used, the measured data is sent directly to the feedback controllers.

vector $y_{m, n}$ contains the measurements at step $n$ and $u_{n-1}$ is the system input as applied in the previous step. The two equations contain only multiplications and additions, and no complex matrix operations such as finding an inverse. Thus, the computations can be performed in a comparably short time.

A key parameter of the Kalman filter is the Kalman gain [29]. Using the system model as described above, the Kalman filter algorithm calculates predictions for the true states of the dynamic system in each execution step. The Kalman gain is then used to weight these predictions relative to the measurements in order to form a weighted estimate of the system states, which in this case, is also the Kalman filter's output. Consequently, this presents a tuning opportunity. The Kalman gain is determined during the design process as optimal solution of the Kalman estimation problem, and affected by the assumed process noise standard deviation $\sigma_{\mathrm{HB}}$ (see (6)) [29], [37]. By increasing this value, the filter increases the weight on the measurements compared to the predictions from its linear model, as there is a high uncertainty in the dynamic system (i.e., increased process noise). Consequently, the noise content of the estimates increases. On the other hand, by choosing smaller values for $\sigma_{\mathrm{HB}}$, which leads to a Kalman gain that weights the measurements less, as the filter then trusts its predictions more, noise in the estimates can be reduced. In summary, the behavior of the Kalman filter can be tuned with the noise covariance matrices, which affect the resulting Kalman gain. As shown in [15], the Kalman gain also affects the linearity of the amplifier due to the utilization of a linear system model in conjunction with a slightly nonlinear physical system. For the demonstrator system, values for $\sigma_{\mathrm{HB}}$ are experimentally determined in order to maximize the achievable SNR, while still obtaining stable Kalman filters. Values between 1 and 15 result, depending on the utilized load configuration.

Fig. 11 illustrates the implementation of the Kalman algorithm and the signal paths inside the demonstrator's Xilinx Zynq Z-7020 processing system. At the beginning of each PWM cycle (if $f_{\mathrm{PWM}}=100 \mathrm{kHz}$, or every second cycle if $f_{\mathrm{PWM}}=200 \mathrm{kHz}$ ), the sensors measure all seven system states and transmit the data to the FPGA fabric, where the raw ADC measurements are rescaled to 32 bit wide fixed-point data vectors that represent the physical quantity in $\mu \mathrm{A}$ or $\mu \mathrm{V}$, which is sufficient to cover 


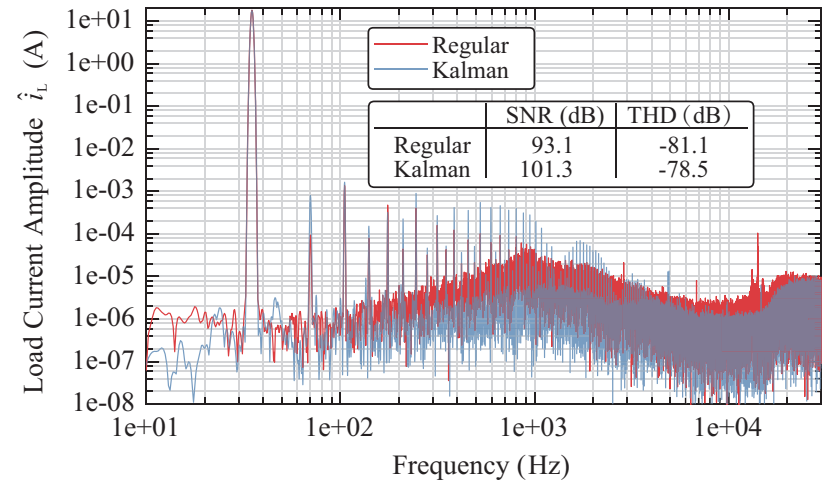

Fig. 12. Spectra of the load current obtained from the amplifier's load current sensor, with and without the use of Kalman estimates for the feedback control system. THD includes the first 9 harmonics and the SNR includes noise from DC to $10 \mathrm{kHz}$. $f_{\mathrm{PWM}}=100 \mathrm{kHz}, u_{\mathrm{DC}}=400 \mathrm{~V}, \hat{i}_{\mathrm{L}}=18 \mathrm{~A}, f_{\mathrm{F}}=35 \mathrm{~Hz}$, Load: $5 \Omega+2.5 \mathrm{mH}$. FFT window: Kaiser, $38^{\text {th }}$ order. Nr. of samples: $20 \mathrm{e} 6$. Sampling rate: $5 \mathrm{MHz}$. Kalman filter executed with $100 \mathrm{kHz}$.

the dynamic ranges of the measurements, while also providing sufficiently low quantization noise. If the Kalman filter is enabled, an interrupt is raised in the CPU and the measurements, together with the previously requested half-bridge voltages $u_{n-1}$, are transferred from the FPGA fabric to the cache of one of the ARM Cortex CPUs. Due to the small data payload (44 bytes), this transfer requires approximately the same amount of time with register-based CPU bus transfers (AXI4-Lite) as with a cache-coherent direct memory access. Next, the transferred data is converted to a floating-point representation and the Kalman filter algorithm is computed, using the processor's floating-point unit, according to (8) and (9). The matrices $A_{k}, B_{k}, C_{k}$ and $D_{k}$ are stored as floating-point arrays in the processor memory. The updated estimates $y_{e}$ of the system states are then transferred back to the FPGA fabric (again, as 32-bit fixed-point numbers), where the cascaded controllers are executed using the estimates instead of the measurements. Subsequently, the delta-sigma noise shapers (NS) and pulse-width modulators are updated with the new duty cycles as requested by the control system, and the process, whose execution requires $\approx 8 \mu \mathrm{s}$, is complete and repeated with the start of the next PWM cycle.

Fig. 12 shows two frequency spectra of $\hat{i}_{\mathrm{L}}$ to demonstrate the influence of the Kalman filter, as the only difference between the two measurements is the utilization of either the Kalman estimates or directly the measurements for the closed-loop control system. As illustrated, the Kalman filter increases the load current SNR significantly, which is also evident from the lower noise floor in the spectrum. However, as explained in [15], the Kalman filter affects THD unfavorably.

Fig. 13 illustrates the SNR improvement of the Kalman filter for the three analyzed loads, with a controller update rate of $f_{\mathrm{PWM}}=100 \mathrm{kHz}$. As observed in Section II, at very high SNR values, the achievable performance is limited by the noise of the DC supply. Nonetheless, the SNR can be improved in excess of $10 \mathrm{~dB}$ by the presented steady-state Kalman filter, which only requires sufficient computational power.

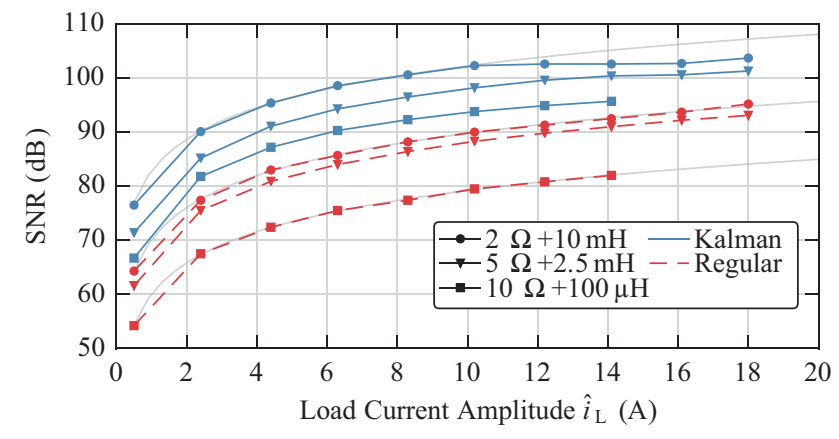

Fig. 13. Measurement of the load current SNR improvement by the Kalman filter. Deviation from fitted line at high SNR is due to DC supply noise at high powers. All controllers and Kalman filter executed with $f_{\mathrm{PWM}}=100 \mathrm{kHz}$. $u_{\mathrm{DC}}=400 \mathrm{~V}, f_{\mathrm{F}}=35 \mathrm{~Hz}$.

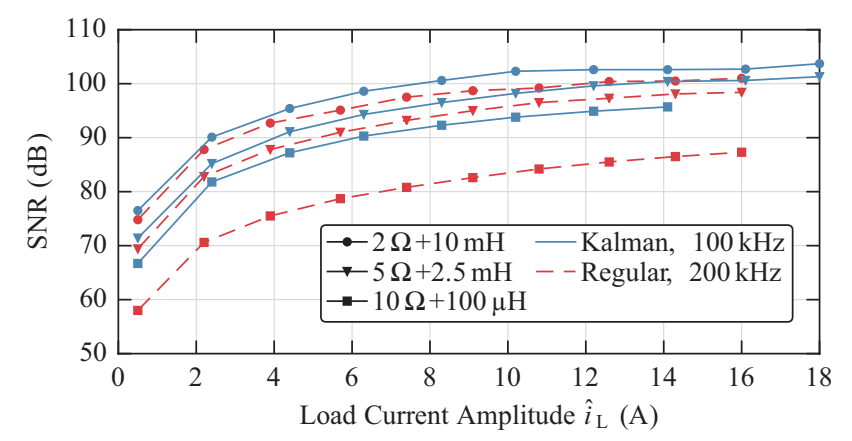

Fig. 14. Load current SNR performance comparison between the control system with the enabled Kalman algorithm, calculated at a rate of $100 \mathrm{kHz}$, and the regular controllers that use the unmodified measurements, but tuned for an execution rate of $200 \mathrm{kHz}$. The PWM frequencies correspond to the controller execution rates. This plot combines the data from Fig. 7 and Fig. 13.

However, despite employing a sophisticated processing platform, the Kalman filter execution rate is limited to $100 \mathrm{kHz}$, which is mainly caused by the time required to perform the floating-point operations for the calculation of (8) and (9). The execution time could be lowered by implementing the algorithm in the FPGA fabric as a digital design. This, however, substantially increases complexity, prolongs development times and reduces design flexibility [29].

A similar noise performance than what is achievable with an enabled Kalman algorithm can also be obtained with the regular control system, but executed with $f_{\mathrm{PWM}}=200 \mathrm{kHz}$. This facilitates controllers with higher open-loop gains, which reduces the influence of noise sources such as the DC supply or the pulse-width modulators. Fig. 14 illustrates this by comparing the relevant measurements. If the Kalman filter would also be executed at a rate of $200 \mathrm{kHz}$, the SNR would even further improve. The Kalman filter is especially effective at improving the SNR of the $100 \mu \mathrm{H}$ load, despite the reduced control gains. This load inductor is not subject to significant external noise due to its few winding turns and small size. Thus, sensor noise is a dominant contributor to the amplifier's overall output noise in this configuration and hence, the Kalman filter can effectively reduce this noise source. Higher control gains can only marginally improve the SNR of this load, as sensor noise dominates (cf. Fig. 7). 
The two other loads generally achieve a better SNR due to their higher impedances, but sensor noise is not dominating, as these inductors are subjected to noise related to external EMI sources (due to their large size and number of winding turns) and hence, the Kalman filter is not as effective.

In summary, the performance gain achievable with the Kalman algorithm depends on the noise environment of the amplifier. If the load current noise is dominated by sensor noise, the Kalman filter can provide a significant improvement. If other sources of noise, which act as disturbances to the control system dominate, it can be advantageous to refrain from utilizing a Kalman filter and instead select a higher controller execution rate. If the increase of $f_{\mathrm{PWM}}$ is not possible due to the resulting increase of converter switching losses, a real-time Kalman filter provides a viable method to eliminate noise in closed-loop converter systems that use digitally acquired measurements. Thus, in order to reduce cost and sensor complexity, more noisy voltage or current sensors could also be utilized, as the measurement noise is effectively filtered by the Kalman estimator.

The implications of the Kalman filter on amplifier linearity are discussed in [15], where it is shown that the Kalman estimator rejects harmonic signal components from the measurements, as the linear system model does not account for nonlinearities that cause distortion (like saturating inductors or the dead time of the bridge legs). A similar effect potentially influences the noise rejection of the amplifier when the Kalman filter is used, as the filter's linear model cannot consider external disturbances, like EMI that couples directly into the load, e.g., into the windings of an actuator. Consequently, such signal components are attenuated by the Kalman estimator and not present in its output. Thus, they do not appear at the inputs of the feedback control system and therefore cannot be rejected. Due to the often parasitic nature of such external noise sources, an application-specific tuning of the Kalman gain is required in order to optimize the overall noise rejection of the amplifier, or an inclusion of such sources, if they can be modeled with sufficient accuracy, in the process noise matrix.

\section{FurTher NoISE SOURCES}

The demonstrator system allows investigating additional sources of amplifier noise. In the following, the benefits of deltasigma noise shapers on the load current noise of the converter operating under closed-loop conditions is demonstrated. Furthermore, it is revealed that the low-distortion dual buck topology does not have any implications on output SNR.

\section{A. Modulator Quantization Noise}

Due to the relatively low-resolution pulse-width modulators often employed in power electronics converters (usually less than 10 bits of amplitude resolution are available for PWM frequencies in excess of $100 \mathrm{kHz}$ ). Therefore, significant wide-band quantization noise is added to the PWM output signals, which is consequently also present in the switched

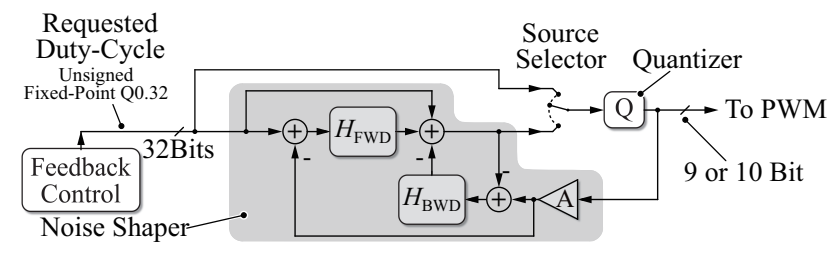

Fig. 15. Noise shaping in the demonstrator system reduces PWM quantization noise from DC to $10 \mathrm{kHz}$. Further details are given in the Appendix. The noise shaper consists mainly of two FIR filters $\mathrm{H}_{\mathrm{FWD} / \mathrm{BWD}}$ [17] and can be bypassed to investigate its effect.

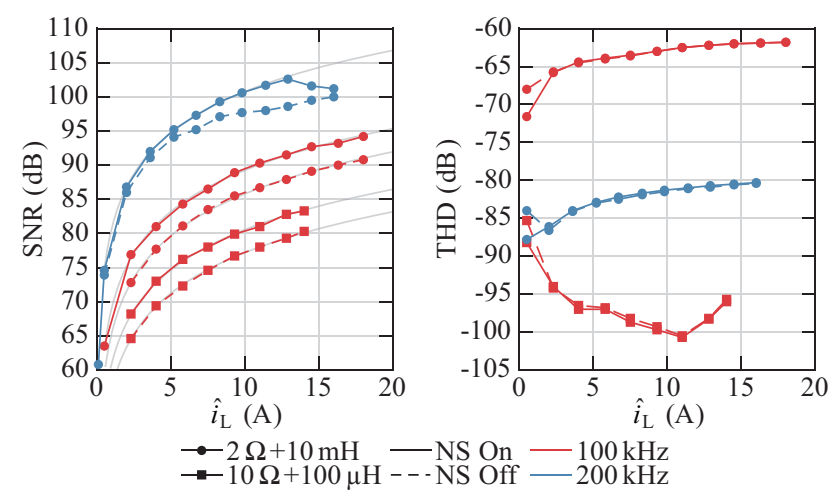

Fig. 16. Experimentally analyzed effects of delta-sigma modulation on SNR with closed-loop converter operation. Regular cascaded control topology (Kalman filter disabled), executed with $f_{\mathrm{PWM}}=100 \mathrm{kHz}$ and $200 \mathrm{kHz}$. At high load currents, the DC supply noise affects SNR. As shown, THD is not affected by the noise shapers. $u_{\mathrm{DC}}=400 \mathrm{~V}, f_{\mathrm{F}}=35 \mathrm{~Hz}$.

half-bridge waveforms [17]. This naturally reduces the load current SNR. Special quantization techniques (delta-sigma modulation/noise shaping) that are capable of creating lownoise (i.e., high-resolution) signals in a certain bandwidths (e.g., from DC to $10 \mathrm{kHz}$ ) are thus highly beneficial for lownoise PWM power amplifiers [38]. The basic functionality of this method is briefly explained in the Appendix. As shown in [17], [18], such noise shaping quantizers are added to the input of each PWM unit, which subsequently is able to provide a low-noise PWM output signal in the desired frequency band. Fig. 15 illustrates the (disengageable) system as used in the demonstrator.

During open-loop operation of the power converter, when no closed-loop feedback system is active, the noise shaper significantly increases the SNR of a pulse-width modulated half-bridge output voltage by up to $30 \mathrm{~dB}$ [17]. Fig. 16 illustrates the influence of the noise shapers on SNR during closed-loop converter operation. With bypassed noise shapers, the regular, truncating quantizer creates significant noise and thus, the load current SNR degrades by up to $5 \mathrm{~dB}$. This effect is less pronounced if the higher-gain control system $\left(f_{\mathrm{PWM}}=200 \mathrm{kHz}\right)$ is utilized, as it is capable of rejecting more quantization noise than the controllers that are executed with $100 \mathrm{kHz}$. Again, at high load powers, the influence of the DC supply becomes visible as it deteriorates the SNR.

In the same measurement, the load current total harmonic 


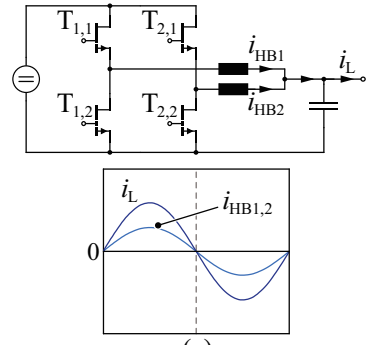

(a)

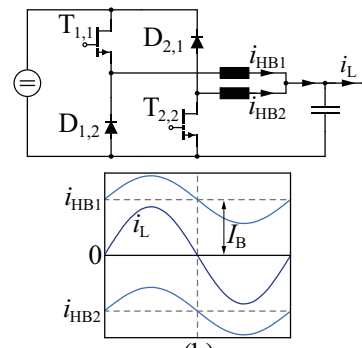

(b)
Fig. 17. (a) Regular interleaved half-bridge power stage and the corresponding current waveforms. (b) Dual buck topology, with unidirectional halfbridge currents. In the demonstrator system, the two power diodes are replaced by identical power transistors [15]. This results in the same power transistor arrangement as shown in (a). Further information is provided in [15].

distortion (THD) is also acquired and it is evident that the noise shaping modulator does not affect amplifier linearity. The distortion measurement method is detailed in [15]. This behavior is expected as the noise shaper only affects quantization noise [17].

A noise shaping modulator is a valuable addition to any lownoise PWM conversion system, as it is capable of attenuating a significant noise source. This is especially relevant for power converters without feedback control systems, as shown in [17], [18]. Nonetheless, the noise shaper benefits are still evident in high-performance closed-loop systems. The implementation of the selected noise shaper only requires two comparably small FIR filters (whose orders are usually smaller than 15) that can conveniently be implemented with an FPGA.

\section{B. Low-Distortion Dual Buck Topology}

Due to the realization of the demonstrator bridge legs with two interleaved half-bridges, two different power stage topologies can be implemented in order to investigate their influence on converter output distortion [15]. Fig. 17 illustrates the regular interleaved half-bridge and the dual buck topology, together with their corresponding waveforms.

In the regular interleaved topology, both half-bridges of each phase conduct an (ideally) identical current, i.e., half the output (load) current. However, this topology is subject to distortion caused by half-bridge dead time. The dual buck topology introduces a circulating current between the two half-bridges of each phase (cf. Fig. 17(b)), which renders the half-bridge currents unidirectional and hence reduces dead time related distortion significantly. A detailed analysis and comparison of these topologies is presented in [15].

The effect of the two power stage topologies on the load current SNR is investigated with the measurements in Fig. 18. The experimental analysis reveals that the load current SNR is neither affected by the power stage topology, the employed dead time or the DC supply voltage. This is expected as dead time is always constant and introduces no possible source of noise. The dual buck behavior of the power converter is achieved by altering the current references of the four halfbridge current controllers such that the desired circulating

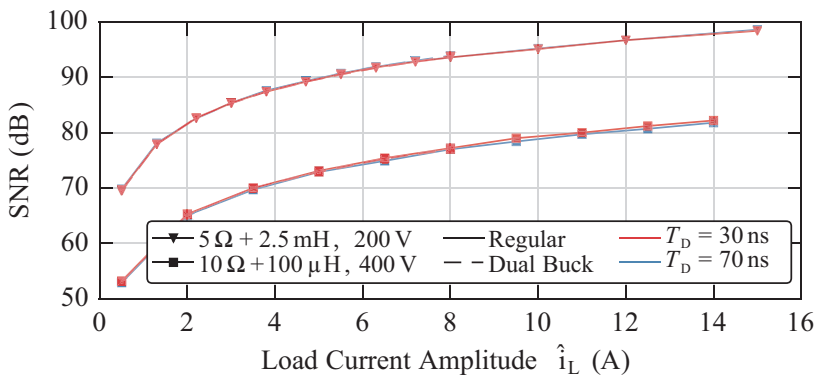

Fig. 18. Experimental analysis of the dependency of load current SNR on halfbridge dead time $T_{\mathrm{D}}$ and power stage topology. $200 \mathrm{~V}$ measurements taken with $f_{\mathrm{PWM}}=200 \mathrm{kHz}, 400 \mathrm{~V}$ measurements with $f_{\mathrm{PWM}}=100 \mathrm{kHz} . f_{\mathrm{F}}=35 \mathrm{~Hz}$. Kalman filter disabled.

current is introduced. This change in power circuit topology does not provide an additional source of noise. Thus, the only significant disadvantage of the dual buck topology are its increased transistor losses (due to the additional circulating current) and hence, a reduced amplifier output current capability [15].

\section{Conclusion}

In this paper, important system constituents for achieving low-noise, high-power output waveforms of digitally controlled switch-mode power amplifiers are presented, analyzed and directly compared. The results are, e.g., relevant for the applications of such systems in nanometer-scale positioning tasks such as lithography processes in integrated circuits manufacturing that require actuator current signal-to-noise ratios (SNR) in excess of $100 \mathrm{~dB}(\mathrm{DC}-10 \mathrm{kHz})$, which is demonstrated in this work. A $400 \mathrm{~V}$ power converter based on gallium nitride $(\mathrm{GaN})$ power transistors is used for demonstrating different influence factors. Further details of the prototype can be found in [15].

First, noise is effectively attenuated by a feedback control system. It is shown how digital controllers with high open-loop gains are enabled by fast execution rates and consequently, high converter PWM frequencies. The demonstrator system can achieve a pulse repetition rate of $200 \mathrm{kHz}$ which, compared to a control system executed with half this rate, increases load current SNR by $\approx 10 \mathrm{~dB}$ to values in excess of $105 \mathrm{~dB}$.

Measurements with the prototype system reveal the importance of a low-noise DC amplifier power supply, as industrial laboratory supplies are not optimized for low-noise voltage outputs and hence, the amplifier load current SNR is limited at high power levels due to noise injected from the DC supply.

Furthermore, the load current SNR can be improved by employing a real-time, steady-state Kalman filter (seven states and four inputs). It effectively attenuates residual sensor noise, to which the feedback control system in principle reacts sensitively. The high processing power provided by the demonstrator's combination of FPGA and procedural CPU (Xilinx Zynq) enables a Kalman filter execution rate of $100 \mathrm{kHz}$, which increases the load current SNR by more than $10 \mathrm{~dB}$, such that it reaches values of more than $108 \mathrm{~dB}$. 
Finally, it is shown how the delta-sigma modulating noise shapers, used to mitigate the quantization noise of the lowresolution PWM modulators, improve the load current SNR by up to $5 \mathrm{~dB}$, while not affecting distortion.

As an outlook, several options are feasible to even further increase the amplifier SNR. First, the careful consideration of the DC supply of such switch-mode power amplifiers, with regard to output voltage noise and control stability, could prevent the injection of noise into the amplifier circuit. This is especially important for single-phase amplifier loads, as they are characterized by a pulsating power flow. A power pulsation buffer could be used to relieve the power supply from delivering a pulsating current and/or to stabilize the amplifier's supply voltage [39]. Additionally, an active power supply filter, basically consisting of a non-isolated DC/DC converter that employs low-noise technologies as presented in this work, could be connected between the (noisy) DC power supply and the precision amplifier, to provide a well-stabilized, low-noise supply voltage.

Another method to increase the load current SNR is offered by paralleling the outputs of $N$ precision amplifiers (e.g., by interleaving more half-bridges). The output noise of the different converters is (ideally) uncorrelated and hence, the overall noise power only increases with $\sqrt{N}$, whereas the available power of the intended output signal increases linearly with $N$, which boosts the achievable SNR [40]. A higher number of interleaved half-bridges could also open the possibility to use only one noise shaper per amplifier phase and operate it at the effective interleaved frequency $N f_{\mathrm{PWM}}$ of the power converter, as opposed to utilizing one noise shaper per half-bridge, operating with $f_{\mathrm{PWM}}$. This increases the modulator's noise rejection capability in the base band, which further reduces PWM quantization noise [17].

In summary, this work presents a fully digital switchmode power amplifier with unprecedented output noise levels, resulting in load current SNR figures in excess of $100 \mathrm{~dB}$ at rated output. The relatively low realization effort of the proposed system encourages the usage of efficient and highpower switch-mode amplifiers in applications that currently rely on complex and low-efficiency linear or hybrid systems.

\section{APPENDIX}

\section{A. Limits of Digital PWM}

Digital PWM is widely adopted for the generation of power switch control signals due to the constant modulation frequency and simple structure. In the presented application, it is used to obtain the half-bridge switch control signals. The modulators are commonly based on digital counters, as illustrated in Fig. 19. It is evidant that each counter step represents a possible PWM duty cycle and hence, the duty cycle (and correspondingly, the half-bridge output voltage) can only take values from a discrete set (i.e., it is quantized), with the number of available counter steps representing the respective duty cycle resolution.

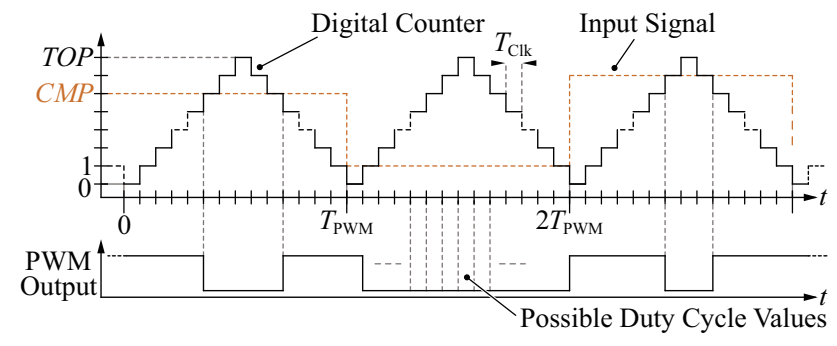

Fig. 19. Digital pulse-width modulation. Each counter step, which, due to its digital nature, is amplitude-quantized, represents a possible duty cycle. Consequently, the duty cycle is also quantized correspondingly, as the halfbridge output voltages can only take values from a discrete set that corresponds to the available duty cycles.

Thus, the achievable SNR of the PWM output signal is limited due to quantization errors that are common to signals of finite amplitude resolution. The SNR of a sinusoidal, amplitude-quantized digital signal (e.g., the modulated halfbridge transistor gate control signal) with a resolution of $n$ bits can be approximated by

$$
\mathrm{SNR} \approx 6.02 n+1.76 \mathrm{~dB} .
$$

The corresponding quantization noise occurs in a frequency range from DC to half the signal's sampling frequency $\left(f_{\mathrm{s}} / 2\right)[20]$.

To obtain low-noise digital signals (e.g., in this case from a PWM modulator), a sufficient number of amplitude levels is mandatory. This requires high digital clock frequencies such that the digital counter covers all required values within the PWM period. For example, to obtain a PWM signal with a pulse repetition rate of $f_{\mathrm{PWM}}=50 \mathrm{kHz}$ and a duty cycle resolution of $n=17$ bits, a clock frequency $f_{\mathrm{Clk}} \approx 13.1 \mathrm{GHz}$ is necessary. However, the physical implementation of high-resolution counters operating at such high clock rates is unfeasible with state-of-the-art digital logic circuits. Consequently, low-SNR PWM signals cannot be generated at PWM frequencies that are common in power electronics (e.g., $\approx 10 \mathrm{kHz}$ to $1000 \mathrm{kHz}$ ) with regular modulation techniques.

\section{B. Delta-Sigma Modulation}

Noise shaping delta-sigma modulation comprises different methods that can alter the frequency distribution of quantization noise [38]. In the context of the presented digital power amplifier, the employed noise shaping modulators shift the quantization noise to frequencies above $10 \mathrm{kHz}$, where it does not affect mechatronic positioning systems. This quantization noise is necessarily generated when lowresolution digital signals are created from high-resolution references, such that regular, low-resolution pulse-width modulators can be employed. Fig. 20 exemplarily illustrates the resulting noise power spectral densities of the signals that are supplied to regular pulse-width modulators. This allows the unaltered replication of the high-resolution reference signals with low-resolution PWM in a certain frequency band. 


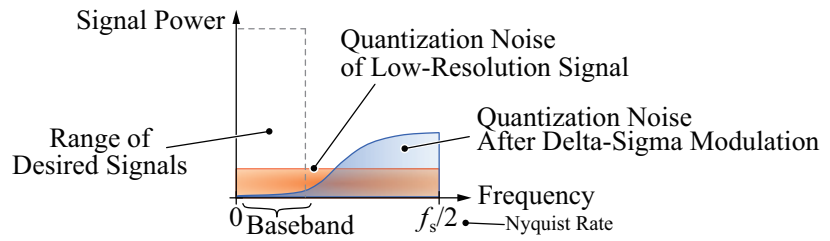

Fig. 20. Illustration of spectral densities of quantization noise of digital, sampled signals (sampling rate $f_{\mathrm{s}}$ ), both with and without delta-sigma modulation. Quantization noise can be shifted to higher frequencies to obtain a low-noise baseband.

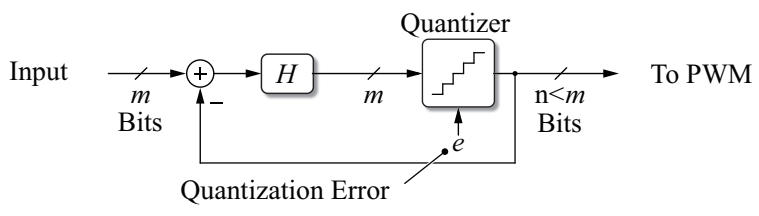

Fig. 21. Simplified noise shaping principle. The transfer function $H$ can be designed to attenuate the quantization noise (caused by the quantization error $e$ ), while minimally affecting the desired signal, in this example.

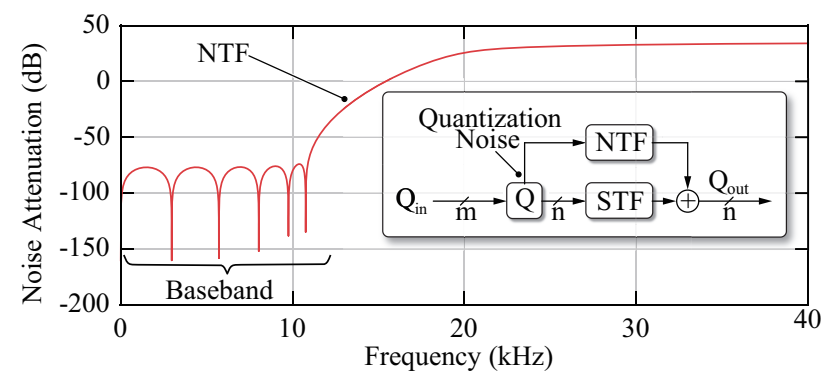

Fig. 22. Amplitude plot of an exemplarily selected noise transfer function (NTF). The high-pass characteristic shifts the quantization noise to higher frequencies. The signal transfer function is usually unity and does not affect the desired signal.

The basic concept behind a noise shaping signal conversion system, that is used to reduce the resolution of digital signals, is illustrated in Fig. 21. The digital reference input signal has a high amplitude resolution of $m$ bits, whereas the lowresolution output ( $n$ bits), which, in the discussed application can be used as input for the subsequent pulse-width modulator, is generated by rescaling and truncating the input signal, which maps it to the (smaller) range of the output signal. The transfer function of this exemplarily selected simple arrangement is given as

$$
\text { Output }=e \cdot \underbrace{\frac{1}{1+H}}_{\text {NTF }}+\text { Input } \cdot \underbrace{\frac{H}{1+H}}_{\text {STF }},
$$

where the noise transfer function (NTF) determines the effect of the quantization noise $e$ on the output, and the signal transfer function (STF) describes the behavior of the input signal on the output. Noise-shaping delta-sigma modulation structures are generally designed such that STF $=1$.

Thus, only the quantization noise is affected by the deltasigma modulator, whereby the NTF is selected such that the noise is shifted to higher frequencies (high-pass characteristic). Fig. 22 illustrates an NTF that attenuates noise in the baseband from $\mathrm{DC}$ to $10 \mathrm{kHz}$ by $\approx 75 \mathrm{~dB}$.

Further details on the specific implementation of the deltasigma modulators utilized in the demonstrator amplifier's control system are given in [17], [18].

\section{REFERENCES}

[1] E. Csencsics, M. Thier, R. Hainisch, and G. Schitter, "System and control design of a voice coil actuated mechanically decoupling twobody vibration isolation system," IEEE/ASME Trans. Mechatronics, vol. 23, no. 1, pp. 321-330, Feb. 2018.

[2] F. Matichard, B. Lantz, K. Mason et al., "Advanced LIGO two-stage twelve-axis vibration isolation and positioning platform. Part 1: Design and production overview," Journal of the International Societies for Precision Engineering and Nanotechnology, vol. 40, pp. 273-286, 2015.

[3] H. Butler, "Position control in lithographic equipment," IEEE Trans. Control Syst., vol. 31, no. 5, pp. 28-47, Oct. 2011

[4] R. Munnig Schmidt, G. Schitter. and J. van Eijk, the Design of High Performance Mechatronics. Delft University Press, 2011, ISBN: 9781-60750-825-0.

[5] T. Oomen, R. van Herpen, S. Quist, M. van de Wal, O. Bosgra, and M. Steinbuch, "Connecting system identification and robust contro for next-generation motion control of a wafer stage," IEEE Trans. Control Syst. Technol., vol. 22, no. 1, pp. 102-118, Jan. 2014.

[6] S. Mirić, A. Tüysüz, and J. W. Kolar, "Comparative evaluation of linear-rotary actuator topologies for highly dynamic applications," in Proc. of the IEEE International Electric Machines and Drives Conference, May 2017, pp. 1-7.

[7] J. van Schoot, K. van Ingen Schenau, C. Valentin, and S. Migura "EUV lithography scanner for sub-8nm resolution," in Proc. of the Society of Photo-Optical Instrumentation Engineers (SPIE), 2015, vol. 9422 .

[8] J. M. Schellekens, H. Huisman, J. L. Duarte, M. A. M. Hendrix, and E. A. Lomonova, "An analysis of the highly linear transfer characteristics of dual-buck converters," IEEE Trans. Ind. Electron., vol. 65, no. 6, pp. 4681-4690, June 2018.

[9] T. Oomen, R. van Herpen, S. Quist, M. van de Wal, O. Bosgra, and M. Steinbuch, "Connecting system identification and robust control for next-generation motion control of a wafer stage," IEEE Trans. Control Syst. Technol., vol. 22, no. 1, pp. 102-118, Jan. 2014.

[10] Y. M. Choi and D. G. Gweon, "A high-precision dual-servo stage using halbach linear active magnetic bearings," IEEE/ASME Trans. Mechatronics, vol. 16, no. 5, pp. 925-931, Oct. 2011.

[11] T. Hu and W. J. Kim, "Extended range six DOF high-precision positioner for wafer processing," IEEE/ASME Trans. Mechatronics, vol. 11, no. 6, pp. 682-689, Dec. 2006.

[12] G. Parmar, K. Barton, and S. Shorya, "Large dynamic range nanopositioning using iterative learning control," Journal of the International Societies for Precision Engineering and Nanotechnology, vol. 38, no. 1, pp. 48-56, 2014.

[13] G. Gong, "Hybrid amplifiers for AC power source applications," Ph.D.dissertation, ETH Zurich, no. 18173, 2009.

[14] H. Ertl, J. W. Kolar, and F. C. Zach, "Basic considerations and topologies of switched-mode assisted linear power amplifiers," IEEE Trans. Ind. Electron., vol. 44, no. 1, pp. 116-123, Feb. 1997.

[15] M. Mauerer and J. W. Kolar, "Distortion minimization for ultra-low THD class-D power amplifiers," CPSS Trans. Power Electron. Appl., 2018.

[16] A. J. Fleming, "Measuring and predicting resolution in nanopositioning systems," Mechatronics, a Journal of the International Federation of Automatic Control (IFAC), vol. 24, no. 6, pp. 605-618, 2014.

[17] M. Mauerer, A. Tüysüz, and J. W. Kolar, "Gate signal jitter elimination and noise shaping modulation for high-SNR class-D power amplifiers," in Proc. of the IEEE Applied Power Electronics Conference and Exposition (APEC), Mar. 2016, pp. 1198-1205.

[18] M. Mauerer, A. Tüysüz, and J. W. Kolar, "Low-jitter GaN E-HEMT 
gate driver with high common-mode voltage transient immunity," IEEE Trans. Ind. Electron., vol. 64, no. 11, pp. 9043-9051, Nov. 2017.

[19] S. S. Saad, "The effects of accumulated timing jitter on some sine wave measurements," IEEE Trans. Instrum. Meas., vol. 44, no. 5, pp. 945-951, Oct. 1995.

[20] W. Kester, the Data Conversion Handbook. Elsevier, 2005, ISBN: 0-7506-7841-0.

[21] J. C. Pedro and N. B. Carvalho, Intermodulation Distortion in Microwave and Wireless Circuits. Artech House, 2003, ISBN: 978-1-58053356-0.

[22] M. Mauerer, A. Tüysüz, and J. W. Kolar, "Voltage/current measurement performance and power supply rejection in all-digital class-d power amplifiers," in Proc. of the Annual Industrial Electronics Society Conference (IECON), Oct. 2016, pp. 666-673.

[23] Xilinx Co., "Zynq-7000 All Programmable SoC," June 2017, Product Specification, no. DS190, rev. 1.11.

[24] LEM Co., "Current Transducer IT 65-S ULTRASTAB," Apr. 2015, Datasheet, no. 88.K7.27.000.0, rev. 1.

[25] Rohde\&Schwarz Co., "UPV Audio Analyzer," Oct. 2015, Manual and Specifications.

[26] S. Skogestad and I. Postlethwaite, Multivariable Feedback Control: Analysis and Design. Wiley, 1996, ISBN: 0-471-94330-4.

[27] R. W. Erickson and D. Maksimović, Fundamentals of Power Electronics, 2nd ed. Springer, 2001, ISBN: 978-0-7923-7270-7.

[28] G. F. Franklin, J. D. Powell, and A. Emami-Naeini, Feedback Control of Dynamic Systems. Pearson, 2010, ISBN: 978-0-13-500150-9.

[29] M. S. Grewal and A. P. Andrews, Kalman Filtering, 4th ed. IEEE Press, 2015, ISBN: 978-1-118-85121-0.

[30] T. Baumgartner and J. W. Kolar, "Multivariable state feedback control of a $500000-\mathrm{r} / \mathrm{min}$ self-bearing permanent-magnet motor," IEEE/ ASME Trans. Mechatronics, vol. 20, no. 3, pp. 1149-1159, June 2015.

[31] B. Bolsens, K. D. Brabandere, J. V. Den Keybus, J. Driesen, and R. Belmans, "Model-based generation of low distortion currents in gridcoupled PWM-inverters using an LCL output filter," IEEE Trans. Power Electron., vol. 21, no. 4, pp. 1032-1040, July 2006.

[32] Young-Real Kim, Seung-Ki Sul, and Min-Ho Park, "Speed sensorless vector control of induction motor using extended kalman filter," IEEE Trans. Ind. Appl., vol. 30, no. 5, pp. 1225-1233, Sept. 1994.

[33] M. A. Eleffendi and C. M. Johnson, "Application of Kalman filter to estimate junction temperature in IGBT power modules," IEEE Trans. Power Electron., vol. 31, no. 2, pp. 1576-1587, Feb. 2016.

[34] V. M. Moreno, A. P. López, and R. I. D. Garcías, "Reference current estimation under distorted line voltage for control of shunt active power filters," IEEE Trans. Power Electron., vol. 19, no. 4, pp. 988 994, July 2004.

[35] M. Ricco, P. Manganiello, E. Monmasson, G. Petrone, and G. Spagnuolo, "FPGA-based implementation of dual kalman filter for PV MPPT applications," IEEE Trans. Ind. Informat., vol. 13, no. 1, pp. 176-185, Feb. 2017.

[36] N. K. Quang, N. T. Hieu and Q. P. Ha, "FPGA-based sensorless PMSM speed control using reduced-order extended Kalman filters," IEEE Trans. Ind. Electron., vol. 61, no. 12, pp. 6574-6582, Dec. 2014.

[37] MathWorks Co., "MATLAB documentation for the kalmd command," [Online]. Available: https://mathworks.com/help/control/ref/kalmd. html.
[38] R. Schreier, S. Pavan, and G. C. Temes, Understanding Delta-Sigma Data Converters, 2nd ed. Wiley-IEEE, 2017, ISBN: 978-1-11925827-8.

[39] D. Neumayr, D. Bortis, and J. W. Kolar, "Ultra-compact power pulsation buffer for single-phase DC/AC converter systems," in Proc. of the IEEE International Power Electronics and Motion Control Conference (IPEMC-ECCE Asia), May 2016, pp. 2732-2741.

[40] C. D. Motchenbacher and J. A. Connelly, Low-Noise Electronic System Design, Wiley, 1993, ISBN: 0-471-57742-1.

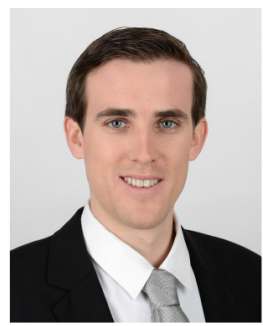

Mario Mauerer received his M.Sc. degree in electrical engineering from ETH Zurich in 2014. During his subsequent Ph.D. studies, whose corresponding degree he obtained in 2018 from the Power Electronic Systems Laboratory, ETH Zurich, he investigated ultra-high precision and high-power wide-bandgap switch-mode power amplifier systems. He is currently working as a postdoctoral scientist at the Power Electronic Systems Laboratory with research topics regarding three-phase rectifiers, precision amplifiers and magnetically levitated actuators.

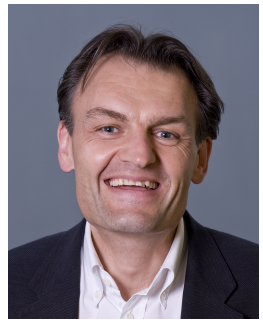

Johann W. Kolar received his M.Sc. and Ph.D degree (summa cum laude / promotio sub auspiciis praesidentis rei publicae) from the University of Technology Vienna, Austria, in 1997 and 1999, respectively. Since 1984 , he has been working as an independent researcher and international consultant in close collaboration with the University of Technology Vienna, in the fields of power electronics, industrial electronics and high performance drives. He has proposed numerous novel PWM converter topologies, and modulation and control concepts and has supervised over $70 \mathrm{Ph}$.D. students. He has published over 650 scientific papers in international journals and conference proceedings, 4 book chapters, and has filed more than 160 patents. The focus of his current research is on ultra-compact and ultra-efficient $\mathrm{SiC}$ and $\mathrm{GaN}$ converter systems, wireless power transfer, solid-state transformers, power supplies on chip, and ultra-high speed and bearingless motors. Dr. Kolar has received 27 IEEE Transactions and Conference Prize Paper Awards, the 2014 IEEE Middlebrook Award, the 2016 IEEE William E. Newell Power Electronics Award, the 2016 IEEE PEMC Council Award and two ETH Zurich Golden Owl Awards for excellence in teaching. He initiated and/or is the founder of four ETH Spinoff companies. He is a member of the steering committees of several leading international conferences in the field and has served from 2001 through 2013 as an Associate Editor of the IEEE Transactions on Power Electronics. Since 2002 he also is an Associate Editor of the Journal of Power Electronics of the Korean Institute of Power Electronics and a member of the Editorial Advisory Board of the IEEJ Transactions on Electrical and Electronic Engineering. 